Editorial

\title{
Cancer therapy: overview of a new era
}

Volume I Issue 2 - 2014

The aim of conventional cancer therapy has always been to achieve control of the cancer by killing or hampering the growth and proliferation of the cancer cell population while trying to minimize the harm to normal cells and tissues. The majority of chemotherapeutic agents have employed nonspecific DNA-damage and toxins affecting actin filaments and microtubules to target malignant cells, an approach that inevitably affects normal cells and induce resistance to drugs in most heavily treated cancers. In many solid tumors, oncogenesis itself is dependent on the acquisition of resistance to DNA damage and to apoptosis, yielding an intrinsic insensitivity to the effects of chemotherapy. Intrinsic resistance and the narrow margin between safety and efficacy, has limited the impact of chemotherapeutic agents in achieving cure or long remissions in advanced malignancies. As a result, innovative therapeutic means are needed.

Genomic medicine has allowed the identification of an important number of genes which, when mutated, drive the process of oncogenesis and malignant growth. Many of these cancer-driver mutations have been used as targets for the development of targeted therapies that have entered clinical trials and, if approved, clinical practice. So far, two different targeting mechanisms are evident and have proven to be effective: one picking on the direct function of an overactive oncogene such HER2 or EGFR, the other exploiting the inherent susceptibilities of loss of function or function deficiency of critical genes such tumor suppressors or BRCA.

Growth signaling in normal cells is a highly regulated process wherein proliferative signals are activated whenever necessary and deactivated when no longer necessary; this tight regulation ensures cell homeostasis. However, in cancer cells this regulation is compromised. One of the fundamental traits of cancer cells is their ability to proliferate without a controlled signaling input. They achieve this in a number of ways: gene amplification, increasing growth factor production, increasing the number of receptors on the cell surface, activating proteins in the downstream signaling pathway, etc. The molecular mechanisms underlying tumorigenesis in the overactive oncogenes appear to be complex and a unified mechanistic model has not emerged. Certain genetically defined cancers are dependent on a single overactive oncogene for their proliferation and survival, a phenomenon known as "oncogene addiction". Various rationales are provided for targeting this category of genetic alteration in cancer therapy.

\author{
Haji Adel Anis \\ Cedars - Jebel Ali International Hospital, UAE
}

Correspondence: Hajj Adel Anis, Medical Oncologist at Cedars - Jebel Ali International Hospital, 9370 Rue Lajeunesse, Montreal, UAE, Tel 438-992-55I6, Email ahaji@dr.com

Received: October 22, 2014 | Published: October 25, 2014
When loss of function or function deficiency mutations drives tumorigenesis, targeting is conceptually more problematic, and this is where synthetic lethality has gained traction. Synthetic lethality describes a genetic interaction in which single-gene defects are compatible with cell viability, but the combination (or synthesis) of gene effects results in cell death. The most promising example of this strategy is the induction of a second molecular defect that specifically interacts with the preexisting mutation and leads to the death of only those cells carrying the mutation, an approach that exploits the inherent susceptibilities of loss function mutations. The first application of a synthetic lethal therapy to reach the clinic was targeting of BRCA1- or BRCA2-deficient tumor cells with PARP inhibitors.

Scientists are looking forward to an era of "personalized therapy" for cancer patients. The ability to determine which tumors have specific mutations (a peculiar biomarker) and then test those cancers against drugs lets scientists figure out which drugs best treat which types of cancer. The one-size-fits all treatment, which does not take into account the tumor mutation, is still used today, but if this revolution goes forward as many suspect, it will be seen in the future as primitive.

\section{Acknowledgments}

None.

\section{Conflicts of interest}

Author declares there are no conflicts of interest. 